\title{
Systems with Exponential Eigenfunctions and Exponential-Input/Constant-Output Operators
}

\author{
Pedro M. Q. Aguiar and Francisco M. Garcia
}

\begin{abstract}
Recently, Vaidyanathan [1] and Ferreira [2] reported on nonlinear systems with exponential eigenfunctions. In this letter, we introduce a larger class of such systems, generated from a special family of exponential-input/constant-output operators that capture exponential signals from general inputs. In particular, we extend the classes of systems with exponential eigenfunctions defined in [1], [2] to include causal nonhomogeneous time-varying systems.
\end{abstract}

Index Terms-Causality, eigenfunctions, exponential-input/constant-output operators, exponentials, homogeneous time-invariant systems, nonhomogeneous systems, nonlinear systems.

\section{INTRODUCTION}

$\mathbf{T}$ HE WELL-known exponential eigenfunction (EE) property of linear time-invariant (LTI) systems extends to homogeneous time-invariant (HTI) systems, as shown in a recent letter [1]. Additionally, that letter raised the challenge of finding larger classes of systems that respond with exponential outputs to exponential inputs. Subsequently, it was pointed out in [2] that a weighted sum of systems with the EE property has also the EE property. In [2], the weights are represented by functionals, i.e., signal-dependent gains, leading to examples of nonhomogeneous and time-varying systems with the EE property. In this letter, we introduce a new class of systems with the EE property, generated from exponential-input/constant-output (EICO) operators. We show that this new class extends those proposed in [1], [2].

To motivate the use of EICO operators, we emphasize that any system generated according to the method proposed in [2] is, in general, not causal. We overcome this limitation by replacing the aforementioned signal-dependent functionals by convenient operators (mappings between signals). These operators produce constant outputs in response to exponential inputs and arbitrary outputs to nonexponential inputs. We will see that a particular structure of EICO operators that capture exponential signals from general inputs, leads to a large variety of systems with the EE property, including causal nonhomogeneous time-varying ones.

Manuscript received December 14, 1999. The associate editor coordinating the review of this manuscript and approving it for publication was Prof. G. Ramponi.

The authors are with Instituto de Sistemas e Robótica (ISR), Instituto Superior Técnico (IST), Lisboa, Portugal (e-mail: aguiar@isr.ist.utl.pt fmg@isr.ist.utl.pt).

Publisher Item Identifier S 1070-9908(00)06514-7.

\section{NONLINEAR SySTEMS WITH EXPONENTIAL EIGENFUNCTIONS}

Reference [1] proves that HTI systems have the EE property and presents the following example of such a system that is not LTI:

$$
y(n)= \begin{cases}\frac{x^{2}(n)}{x(n-1)}, & \text { if } x(n-1) \neq 0 \\ 0, & \text { if } x(n-1)=0 .\end{cases}
$$

In fact, the system (1) is nonlinear, and its response to an exponential input $x(n)=a^{n}$ is the same exponential multiplied by a complex constant $y(n)=a a^{n}$, i.e., it has the EE property.

More recently, reference [2] proposed a larger class of systems $S$ with the EE property defined by

$$
S[x]=\sum_{i \in J} F_{i}[x] H_{i} x
$$

where $H_{i}$ represents an HTI system, and $F_{i}$ is a functional that maps the input signal into a complex value. We use a notation suggested by [2]: the arguments of nonlinear functionals or systems are written inside square brackets, but the brackets are dropped if the mapping is homogeneous. The rationale behind expression (2) is that a system does not lose the EE property when its output is multiplied by an input-dependent gain. These gains (i.e., the functionals $\left\{F_{i}\right\}$ ) induce possible nonhomogeneous and/or time-varying behaviors for the system $S$. As noted in [2], the systems $\left\{H_{i}\right\}$ need not to be HTI, they may be any systems with the EE property.

As an example of a nonhomogeneous and time-varying system that has the EE property, reference [2] takes the sum (2) to have only one term and specifies $F[x]=1+x(0)$. We observe that the resulting system is not causal because the output at any negative time instant depends on the future value of the input $x(0)$. In fact, all systems generated by (2) are not causal, except in two trivial cases. First, when the functionals $\left\{F_{i}\right\}$ do not depend on the input signal $x$, i.e., they are constants $F_{i}[x]=f_{i}$. Since any input-dependent functional $F_{i}$ is a function of, at least, the value of the input at a specific time instant $x\left(n_{0}\right)$, the output $y(n)$ of the system $S$, for $n<n_{0}$, depends on a future value of the input. Second, when the signals are defined on a half-line $n \geq n_{0}$, and the functionals $\left\{F_{i}\right\}$ depend only on the value of the input signal at the initial time instant $n_{0}, F_{i}[x]=f_{i}\left(x\left(n_{0}\right)\right)$.

\section{EXPONENTIAL-INPUT/CONSTANT-OUTPUT OPERATORS}

In this section, we extend the class of systems with the EE property by replacing the functionals $\left\{F_{i}\right\}$ in (2) with operators 
$\left\{T_{i}\right\}$ restricted to respond with constants to exponential inputs. Thus, we propose the class of systems $S$ defined by

$$
S[x]=\sum_{i \in J} T_{i}[x] H_{i} x
$$

where $H_{i}$ represents an HTI system. We call $T_{i}$ an exponential-input/constant-output (EICO) operator because it maps exponential signals to complex constants

$$
T_{i}\left[a^{n}\right]=t_{i}(a), \quad a \neq 0 .
$$

To show that the class of systems $S$ given by (3) with $T_{i}$ defined by (4) has the EE property, let $x(n)=a^{n}, a \neq 0$. From (4), $T_{i}\left[a^{n}\right]=t_{i}(a)$. Since $H_{i}$ is homogeneous, $H_{i} a^{n}=$ $h_{i}(a) a^{n}$, and the output of the system is the exponential input up to a complex scale factor $y(n)=\left(\sum_{i} t_{i}(a) h_{i}(a)\right) a^{n}$. When $a=0, x(n)=0,\left(H_{i} x\right)(n)=0$, and $y(n)=0$, independently of the operators $\left\{T_{i}\right\}$.

Obviously, the class of systems defined in (3) includes all the systems with the EE property proposed in [1], [2]. The relevant question is whether there are nonconstant operators that satisfy (4). The answer is affirmative. Next, we propose a simple way to generate such EICO operators. By using these operators, we design a wide class of systems with the EE property. Furthermore, unlike the systems generated according to (2), the proposed class includes time-varying, nonhomogeneous, causal systems. To construct such systems, we exploit an operator based on the nonlinear HTI system (1) proposed in [1], which has the capability of capturing exponential signals from general inputs.

The nonlinear system (1) is rewritten as $V[x] x$ according to the structure of (3), where $V[x]$ is the operator defined by

$$
y=V[x] \Leftrightarrow y(n)= \begin{cases}\frac{x(n)}{x(n-1)}, & \text { if } x(n-1) \neq 0 \\ 0, & \text { if } x(n-1)=0 .\end{cases}
$$

From this definition, we see that $V\left[a^{n}\right]=a$. Thus, $V$ is an EICO operator, see (4), and in addition, it exhibits the useful property

$$
V\left[V\left[a^{n}\right]\right]=1, \quad a \neq 0 .
$$

According to (6), $V$ is a particular operator that returns 1 when applied twice to any exponential signal $a^{n}, a \neq 0$.

Using the operator $V$, we define a broad class of EICO operators by

$$
\tilde{T}[x]=(1-V[V[x]]) G[x]+f(V[x](n))
$$

where

$V[x](n)$ response of $V$ to the input signal $x$, at time $n$;

$G \quad$ operator;

$f \quad$ function, i.e., a mapping between complex numbers ( $f$ can also be regarded as a time-invariant memoryless operator acting on $V[x])$.

Due to property (6), the first term of (7) is zero when the input is an exponential $a^{n}, a \neq 0$. As a consequence, $\tilde{T}\left[a^{n}\right]=f(a), a \neq 0$, thus $\tilde{T}$ is an EICO operator for any choice of $G$ and $f$. The complex value $f(a)$ is the gain associ- ated to the exponential input $a^{n}, a \neq 0$. When the input $x$ is not an exponential, it is possible to generate a wide class of output signals by specifying any mapping $G$ in the first term of (7).

As an example of a system constructed with the operator $\tilde{T}$, we take the sum (3) to have only one term and the corresponding HTI system to be the identity operator. By choosing $G$ in (7) to be the time-varying and causal operator $G[x]=n$, we obtain the following input-output relation for the system $\tilde{T}[x] x$ :

$$
y(n)= \begin{cases}\left(x(n)-\frac{x^{2}(n) x(n-2)}{x^{2}(n-1)}\right) n+ & \\ f\left(\frac{x(n)}{x(n-1)}\right) x(n), & \text { if } x(n-1) \neq 0 \\ (n+f(0)) x(n), & \text { if } x(n-1)=0 .\end{cases}
$$

This system has the EE property $\left[x(n)=a^{n} \Rightarrow y(n)=\right.$ $\left.f(a) a^{n}\right]$. Furthermore, it is time-varying, nonhomogeneous, and the output at a given time instant does not depend on future values of the input, i.e., the system is causal.

\section{REMARK}

In the DSP community, an exponential signal is commonly defined by $x(n)=c a^{n}$, see [3]. Such signal is an eigenfunction of any system described by (3) when the EICO operators $T_{i}$ take the form (7). In fact, the EICO operator given by (7) satisfies $\tilde{T}\left[c a^{n}\right]=f(a), a \neq 0$, since, from (5), $V\left[c a^{n}\right]=V\left[a^{n}\right]=a$. As a final remark, we point out that, in opposition to homogeneous systems, nonhomogeneous ones without the EE property may still admit eigenfunctions $a^{n}, \forall a$, i.e., exponentials with $c=1$ in the definition of [3]. An illustrative example follows.

Consider the system $Q[x]=L[x] x$ where the operator $L$ is defined by

$$
y=L[x] \quad \Leftrightarrow \quad y(n)= \begin{cases}\frac{\log x(n)}{n}, & \text { if } n \neq 0 \\ \log x(1), & \text { if } n=0 .\end{cases}
$$

From (9), $L\left[a^{n}\right]=\log a$. Thus, the system $Q$ has eigenfunctions $a^{n}, \forall a$

$$
Q\left[a^{n}\right]=\log a a^{n}
$$

However, the response of $Q$ to an exponential input signal that is exponential up to a scale factor $x(n)=c a^{n}, c \neq 1$ is not exponential. In fact, using (9), we obtain for $n \neq 0$

$$
Q\left[c a^{n}\right]=\log a a^{n}+\log c \frac{a^{n}}{n} .
$$

The previous example shows that some care must be taken when defining eigenfunctions of nonhomogeneous systems.

\section{REFERENCES}

[1] P. P. Vaidyanathan, "Homogeneous time-invariant systems," IEEE Signal Processing Lett., vol. 6, pp. 76-77, Apr. 1999.

[2] P. J. S. G. Ferreira, "Nonlinear systems and exponential eigenfunctions," IEEE Signal Processing Lett., vol. 6, pp. 287-289, Nov. 1999.

[3] A. V. Oppenheim, A. S. Willsky, and W. T. Young, Signals and Systems. Englewood Cliffs, NJ: Prentice-Hall, 1983. 